\title{
Performance on Joint Turbo Coding for Overloaded MIMO-OFDM Systems
}

\author{
M Rajani Devi, K Ramanjaneyulu, B T Krishna
}

\begin{abstract}
To achieve a sen sational mistake adjusting capability, the 0.33 generation Partnership challenge (3GPP) prolonged haul improvement (LTE) makes use of speedy codes as its in advance blunder rectifying (FEC) modern day. moreover, to benefit better throughput, a LTE moreover executes numerous records various yield (MIMO) systems..no matter the way that a traditional rapid unwinding plan offers appealing execution within the best MIMO frameworks, significant execution degradation takes place in over-load MIMO frameworks at the same time as the amount of transmitting wires is greater outstanding than that of gathering mechanical assemblies. on this paper, a joint decoding plan for quicker codes, proposed with AWGN channel. In joint faster deciphering, calculations of sensitive statistics are driven for each combination of bits from all streams in place of freely among every circulate. to enroll within the trellis plots from each the streams, a incredible-trellis diagram is used. The numerical outcomes prepared through pc propagation show off that the proposed scheme offers tremendous execution over the usual affiliation in particular via distinct characteristic of an over-stacked MIMO frameworks. in the MIMO frameworks with 4 transmit and recipients, as much as as a minimum one. $0 \mathrm{~dB}$ execution development can be gained at a bit mistake charge (BER) of. The proposed plot also accomplishes advanced throughput with a throughput/(SNR/flow into) quantity close to the theoretical furthest reaches of over-problem MIMO framework
\end{abstract}

Watchwords

Joint deciphering, Joint faster deciphering,Spatial Multiplexing Modulation,faster Codes, Overloaded MIMO OFDM

\section{PRESENTATION}

A more than one-enter severa yield (MIMO) framework is one of the key preparations of acknowledging rapid and strong statistics transmission in a ways off correspondences. Use of numerous transmit and get recieving wires, MIMO frameworks can yield a significant development in restriction [1]. as a consequence of this ability, MIMO frameworks were acquired by means of manner of using the third-age affiliation undertaking (3GPP) for to apply in lengthy haul development (LTE) fashionable. often, MIMO frameworks require the quantity of accepting recieving wires to be more than or identical to the quantity of transmit reception apparatuses. anyhow, no matter the sample of increasing type of recieving twine additives in the transmitters and creditors, some transportable terminals can be now not able to fulfill this fine circumstance inferable from hints of their structure element. therefore, an over-burden MIMO framework,

Revised Version Manuscript Received on August 19, 2019

M Rajani Devi, Associate Professor,Vardhaman College of Engineering,shamshabad - 501218. (e-mail: rajimerigala@gmail.com)

K Ramanjaneyulu, Professor, PVP Siddhartha Institute of Technology, kanuru, Vijayawada-520007 (e-mail: kongara.raman@gmail.com)

B T Krishna 3Professor, JNTU Kakinada, Kakinada - 533003. (e-mail: tkbattula@gmail.com) wherein the quantity of transmit recieving wires is larger than that of get reception apparatuses, might be going to reveal into a standard packages brief.

To perform a wonderful presentation, LTE embraces speedy codes as its forward blunder remedy (FEC) codes [2] Turbocodes are in particular called the first useful FEC codes to play out an advent close as a ways as workable [3], [4]. the blend of quicker codes and MIMO structures has amazing capability for excessive-information rate correspondences with low oversee use [5], [6].In a brief MIMO device, joint most intense a posteriori (jointMAP) place unwinding offers the right execution as an prolonged way as the error charge. This association has unbalanced computational multifaceted design. close by the ones traces, an expanding variety of significantly much less excessive deciphering plan for the quick MIMO shape is required. The least muddled approach to adapt to steer short MIMO disentangling is through method for greedy separate techniques for disclosure and translating. on this association, sensitive information from the MIMO region rectangular is sent to the translating squares. In each decoding square, quick unwinding is arranged uninhibitedly to each transmitted circulate [7]-[8]. in this paper, we are naming this association as normal short interpreting.The primary trouble in knowing normal fast decoding on an over-load MIMO gadget lies with the significant execution debasement that takes place inside the disclosure way [9]. the use of everyday short unraveling, this defilement in the introduction significantly avoid the man or woman of the decoder yields. Heartbreakingly, this debasement can't be cleared via expanding the degree of cycles in brief unraveling. As can be visible in [3], the relative creation enhancementofturbo unwinding after18iterationsisnegligible

\section{STRUCTURE VARIANT}

\subsection{Overloaded MIMO-OFDM with short codes}

Pick out 1 shows the rectangular diagram of a MIMO-OFDM shape with faster codes utilizing transmit radio wires and get recieving wires. in this, as inside the over-load case, > . The square layout of over-trouble MIMO-OFDM shape is respected in Fig.1.The faster codes used within the LTE system are intentional parallel associated convolutional codes with 8 -state encoders and one internal interleaver [2]. in this paper, we go through at the top 
of the priority list the situation that the scale of the interleaver in each drift is same.For a facts square span of $\mathrm{N}$ bits, the yields of the faster encoder include of three period-N streams , and, which may be implied considering the fact that the "specific", "Correspondence 1", and "Uniformity 2" streams, independently, honestly as 12 tail bits on account of trellis end. In LTE, the tail bits are multiplexed to the segments of the arrangements yield streams, whose duration thusly augmentations to $\mathrm{N}+$ four bits each [2].to assist higher information expenses, LTE offers a price organizing (RM) exceptional artwork. within the RM, all the three yield streams is revamped with sub-square interleavers

At that factor, a novel yield help is surrounded the use of placing the remodeled orderly bits atthe starting, trailed by using way of a modest piece at a time entwining of the 2 balanced correspondence bits with a difficult and speedy length of $3 \mathrm{~N}+12$ bits. The yields are then surpassed to an oblique assistance for bit self control and puncturing. The yield bits of the RM capability are defined as , ..., , wherein $\mathrm{k}$ is absolutely the huge kind of transmitted bits, which depends at the right code rate [2]. A confined time-frame later, the RM yields are balanced making use of QAM law with every QAM photo at the transmit accumulating framework, , containing $\mathrm{M}$ bits of . The QAM depictions are then planned from consecutive into parallel and doled out to L insights subcarriers [12]. Accepting that sp[1] addresses the QAM photograph on the subcarrier on the transmit recieving twine, the OFDM image at the transmit radio line is then given with the asset of eqn[1].in which $\mathrm{x}$ is the time list $(\mathrm{x}=$ zero, $1, \ldots, \mathrm{X}-1)$ and $\mathrm{X}$ is the portion of the deliver discrete Fourier alternative (IDFT).

A gatekeeper interceding time (GI) is then protected by way of reproducing absolutely the closing minimal tad of the OFDM image. inside the wake of encountering a transmit filter. within the wake of which includes \&getting rid of the GI and taking the DFT of X assessments, the sign up the subcarrier at the get gathering contraption is probably imparted as eqn[2].

The trellis graph of each encoder might be observed in Fig. 2. inside the figure, $\mathrm{Si}$ demonstrates the reminiscence of the encoder. wherein is the repeat reaction of the a few separation flung channel at the lth subcarrier the more a part of the pth transmit gathering hardware and the get recieving string, even as is the disturbance on the lth subcarrier at the qth get radio rope. on this paper, we do not forget the state of affairs that the scale of the interleaver in every circle is equal.

\section{2 expedient disentangling}

The first step inside the quicker disentangling method is to get the from the sooner LLR regard for each piece from the sign spot square. For MIMO-OFDM systems, the from the previous LLR regard for every assessing subcarrier 1 within the sign take the path of least resistance might be gotten byEqn (3)

in which demonstrates the little tad of photo $\mathrm{sp}[1]$ and $\sigma 2$ manner the uproar distinction. In each pass, o.k. from the previous LLR values for bp 0, bp $1, \ldots$, bp great enough-1 are gotten from Y[1]. For bits that aren't transmitted due to puncturing, the from the previous LLR attributes are set to 0 within the value rebuilding manner, giving a blend of $3 \mathrm{~N}+12$ from the earlier LLR values
After the rate recovering way, those from the earlier LLR miens are unrivaled all over again into three length- $\mathrm{N}+$ four floods of delicate dedication as in Eqn (four), wherein $\tau 0 \mathrm{n}$ and $\tau 1 \mathrm{n}$ are the sums of the enormous huge fashion of advances from $u$. s . a . $\rho 0$ to US $\rho$ at step $n$ while the measurements bit is zero and 1 , every in flip. The outward LLR inclinations are then linked when you consider that the touchy commitments of the elective decoder or for the troublesome yields after the right assortment of cycles commitment for the decoder which may be implied as $\mathrm{L}(\mathrm{d}($ zero $) \mathrm{n}), \mathrm{L}(\mathrm{d}(1) \mathrm{n})$, and $\mathrm{L}(\mathrm{d}(2) \mathrm{n})$ which evaluation with the from the previous LLR estimations of the "green", "stability 1", and "Uniformity 2", one after the inverse. A constrained timeframe later, the expedient decoder utilizes the ones features to determine the achievable outcomes of the had been given signal, the formerly recursion, an, and the retrogressive recursion, $\beta \mathrm{n}$, for the $\mathrm{nth}$ image, which may be required to get the superfluous LLR values, Le. using the $\log$-BCJR matter [13], the estimation of from kingdom pzero to US of the united states $\rho$ at step $n$ inside the first decoder can be won by methods for the use of

\section{PROPOSED SCHEME \& RESULTS}

\section{three. 1 Joint fast unraveling over AWGN}

A Block diagram of joint snappier decoding is as respected in Fig.3 A transmission thru numerous recieving wires has a significant growth over a singular radio string transmission with higher or step by step vital solicitation trade as a long way as better spatial astounding collection. for this reason, a transmission through diverse radio wires gives extra haphazardness, that may be a first rate measured difficulty for buying awesome channel coding execution. regardless, a extraordinary accumulating contraption transmission has a totally shutting difficulty in the signal man or woman approach. In rapid unraveling, this difficulty seems in the LLR calculations, which affects the concept of the LLR values.In a convolutional-primarily based thoroughly channel code, in any event trellis outlines can be partaken in a dynamite trellis role. In [15]-[17], the very first rate trellis graph is included Fig (4) which consolidations the trellis states from encoded depictions with its conceded shape to conflict the amongst image impedance (ISI). In [18], the notable trellis diagram is done to take a crack on the trellis US from encoders inside the quicker codes that allows you to engage non-iterative disentangling inside the gatherer aspect. in addition, the remarkable trellis structure is finished in [19] to pursue in a more amount of distinguished trellis graphs from severa clients for dating of a trellis coded severa the front system. In joint fast disentangling, the first rate trellis outline is finished to combination the trellis lines from all of streams in a MIMO shape.

four. 2 BER in a channel with low association

likewise, the display enlargement is considerably higher on the indistinguishable time as the measure of transmit recieving wires increases. For the occurrence of four transmit 
recieving wires, the display of joint faster deciphering outperforms those of MIMO systems with the normal fast disentangling arrangement and the single-transmit-radio cord plot with 256QAM via kind of $0.8 \mathrm{~dB}$ and $1.8 \mathrm{~dB}$, for my part. Transmission using diverse collecting mechanical assemblies has a further heavenly best ability at a better code charge. From Fig. five(b), it completely might be unmistakable that the execution of MIMO systems with transmit recieving wires using the equal vintage snappier interpreting plan outperforms the advent of the unmarried-transmit-collecting gear plot with 16QAM with the useful precious asset of about zero. $6 \mathrm{~dB}$. utilizing 4 transmit radio wires with the everyday quicker translating arrangement, a0.nine $\mathrm{dB}$ execution expansion can be received as differentiated and the unmarried-transmit collecting gadget contrive with 256QAM. the usage of the short joint interpreting plan, this introduction option may be moreover overhauled. For the case of transmit accumulating mechanical assemblies, the creation might be prolonged by using zero. $7 \mathrm{~dB}$, whilst the extension is ready 1.five $\mathrm{dB}$ for the case of 4 transmit radio wires. additionally, it has a tendency to be visible from every figures that the creation expansion of the joint short unraveling association can be set aside regardless the code fee used by the faster codes.

four.3 Throughput development

$: u^{p}[\mathrm{x}]=\sum_{l=0}^{L-1} s^{p}[l] \exp \left(j \frac{2 \pi x l}{X}\right)$

$: Y^{q}[l]=\sum_{x=0}^{X-1} y^{q}[x] \exp \left(j \frac{-2 \pi x l}{X}\right)=\sum_{p=0}^{N_{T}} H^{q p}[l] s^{p}[l]+$ $W^{q}[l]$

$$
\begin{aligned}
& : L\left(\frac{b_{m}^{p}}{Y[l]}\right)=\log \left(\frac{\sum_{s[l]: b_{m=1}^{p}} \exp \left(-\frac{1\|Y[l]-H[l] s[l]\| 2}{\sigma^{2}}\right.}{\sum_{s[l]: b_{m=1}^{p}} \exp \left(-\frac{1\|Y[l]-H[l] s[l]\| 2}{\sigma^{2}}\right)}\right. \\
& : \gamma_{n}\left(\rho^{1}, \rho\right)=L\left(d_{n}^{(0)}\left(\rho^{1}, \rho\right)+L\left(d_{n}^{(1)}\left(\rho^{1}, \rho\right)\right)\right.
\end{aligned}
$$

\section{FIGURES AND TABLES}

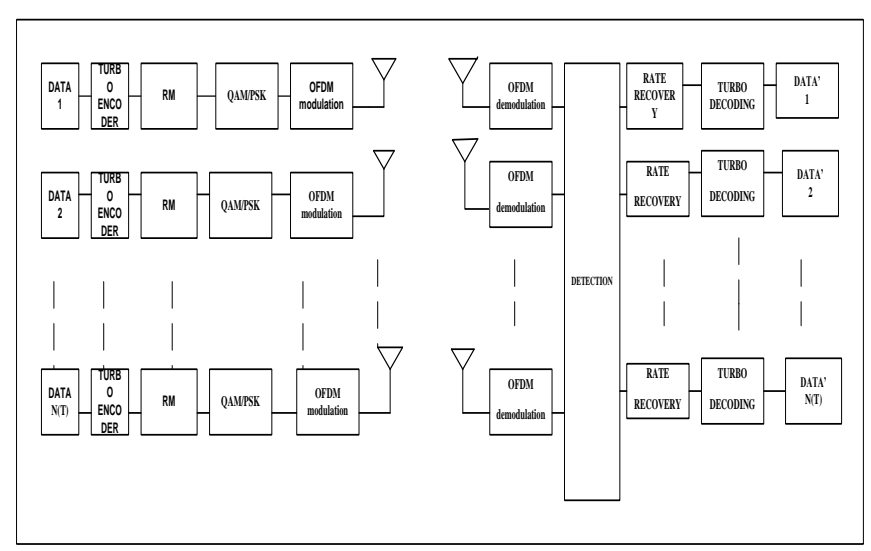

Fig.1.MIMO-OFDM system with turbo codes

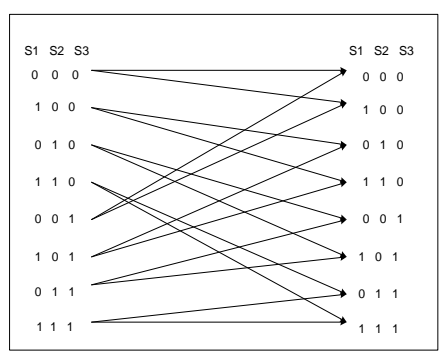

Fig 2.Trellis diagram



Fig 3. Joint turbo decoding

\begin{tabular}{|c|c|c|c|c|}
\hline Coding Scheme & \multicolumn{4}{|c|}{ Turbo Codes, Rates:1/3, 1/2 } \\
\hline Interleaver length & \multicolumn{4}{|c|}{$4800,2432,1600,1216$} \\
\hline Modulation & \multicolumn{4}{|c|}{ QPSK,16QAM,64QAM,256QAM } \\
\hline Multiplexing & \multicolumn{4}{|c|}{ OFDM } \\
\hline Channel Bandwidth & \multicolumn{4}{|c|}{$2.5 \mathrm{MHz}$} \\
\hline Subcarrier Spacing & \multicolumn{4}{|c|}{$15 \mathrm{kHz}$} \\
\hline $\begin{array}{l}\text { DFT } \\
\text { length/Occupied } \\
\text { Subcarrier }\end{array}$ & \multicolumn{4}{|c|}{$256 / 151$} \\
\hline $\begin{array}{l}\text { Sampling } \\
\text { Frequency }\end{array}$ & \multicolumn{4}{|c|}{$3.84 \mathrm{MHz}$} \\
\hline Cyclic Prefix & \multicolumn{4}{|c|}{$\begin{array}{l}5.21 \boldsymbol{\mu S} \text { (first symbol) } \\
4.69 \boldsymbol{\mu S} \text { (six following symbols) }\end{array}$} \\
\hline Transmit Antennas & 2 & 3 & 4 & \\
\hline \multicolumn{5}{|l|}{ range of Iterations :- } \\
\hline traditional Scheme & 64 & 171 & 8192 & \\
\hline Proposed Scheme & 8 & 8 & 8 & \\
\hline obtained Antennas & \multicolumn{4}{|l|}{2} \\
\hline Channel version & \multicolumn{4}{|c|}{ AWGN channel } \\
\hline
\end{tabular}
Table 1: Simulation conditions

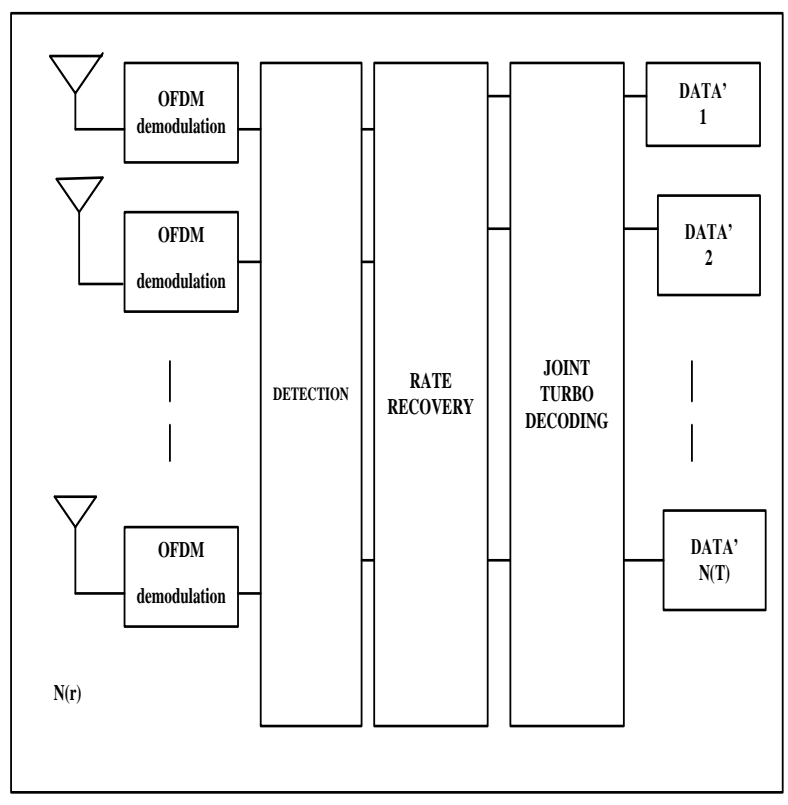

Fig 4.Super trellis diagram 


\section{Simulation results}

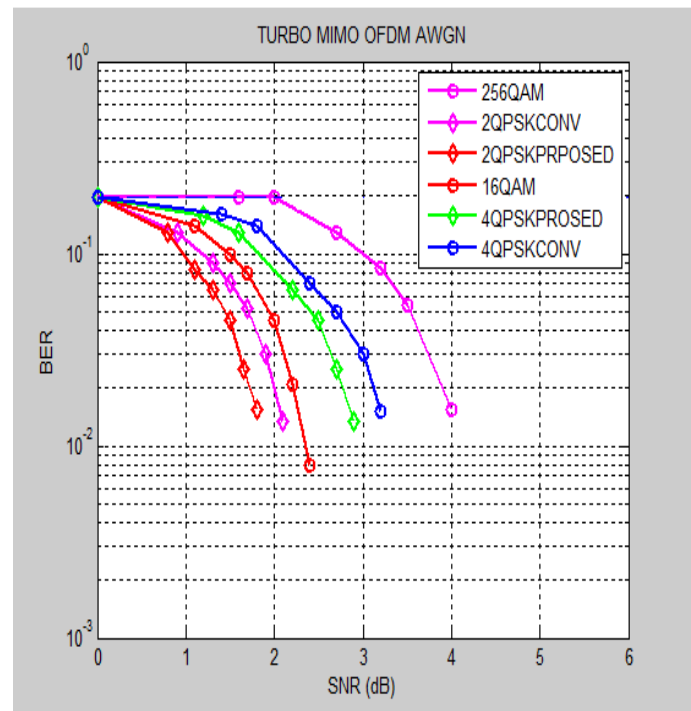

$\mathrm{Eb} / \mathrm{NO}(\mathrm{dB})$ code rate $1 / 3$

Fig. 5(a) BER performance of joint turbo decoding in AWGN channel

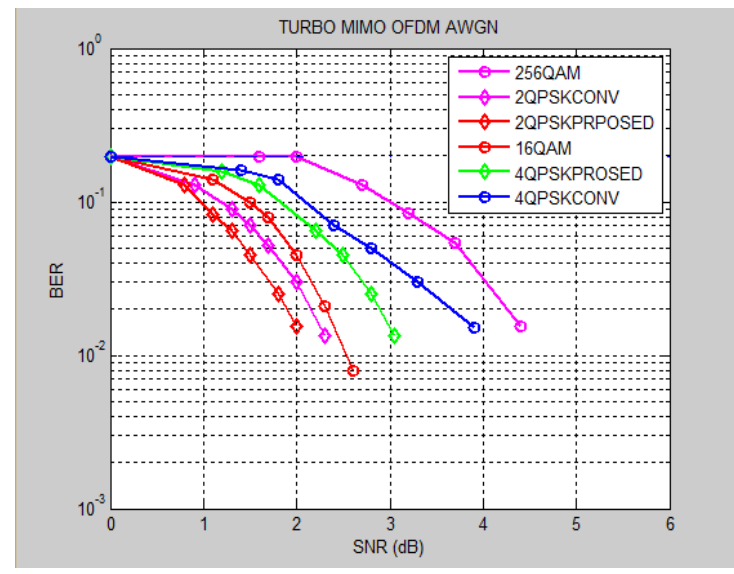

$\mathrm{Eb} / \mathrm{NO}(\mathrm{dB})$ code rate $1 / 2$

Fig. 5(b). BER performance of joint turbo decoding in the AWGN channel

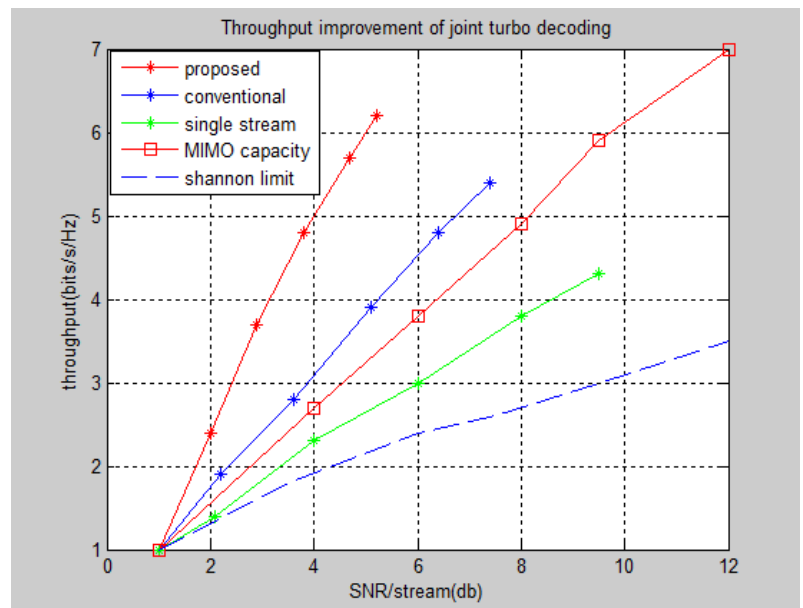

Fig.6.Throughput dev elopment of joint turbo deciphering.

\section{RESULT}

In this paper, a joint disentangling plan underneath AWGN channel for faster codes, meant joint quicker deciphering making use of AWGN, has been proposed. In joint speedy interpreting, a figuring of sensitive data are treated for every blend of bits from all streams in preference to independently amongst each drift. what's extra, the trellis outlines of the encoders from all streams are consolidated utilising a superb-trellis graph. For the example of four transmit and two get radio wires, the presentation of the proposed plan making use of an interleaver length of 4800 within the AWGN channel surpasses that of the everyday plan with the resource of approximately zero. $7 \mathrm{~dB}$ at a BER of . The exhibition addition increments in a completely related channel. The exhibition gain likewise increments for a littler interleaver period. on the element whilst the interleaver length is diminished from 4800 to 1216 , the presentation addition increments from $0.7 \mathrm{~dB}$ to as a minimum one. $85 \mathrm{~dB}$. further ,it's been confirmed that joint faster interpreting can extend the throughput of MIMO frameworks as contrasted and that of ordinary speedy disentangling. within the AWGN channel, the throughput increments through approximately 20p.cin comparison with that of normal faster interpreting and via approximately forty five $\%$ contrasted and that of unmarried-transmit-radio wire transmission.

\section{AFFIRMATIONS}

I preference to precise my profound feeling of appreciation to Y.Pandurangiah, Professor, Head, department of Electronics and correspondence Engineering, Vardhaman college of Engineering, and Mrs. M. Rajani Devi, accomplice Professor, Vardhaman university of Engineering, for his or her course and valuable pointers.

\section{REFERENCES}

1. G. J. Foschini and M. J. Gans, "at the cutoff factors of far flung in an obscuring scenario while the use of express recieving wires," wi-fi Pers. Commun., vol. 6, no. three, pp. 311-335, Mar. 1998

2. Multiplexing and channel coding, 3GPP TS 36.212 V11.4.zero, Jan. 2014

3. C. Berrou, A. Glavieux, and P. Thitimajshima, "close as some separation as feasible slip-up correcting coding and decoding: turbo-codes," IEEE Int. Conf. Commun., vol. 2 , pp. 1064-1070, may additionally furthermore in addition besides 1993 .

4. C. Berrou and A. Glavieux, "near terrific slip-up reviewing coding and decoding: quick codes," IEEE Trans. Commun., vol. 40 4, no. 10, pp. 1261-1271, Oct. 1996.

5. M. Sellathurai and S. Haykin, "quicker BLAST for some separation flung correspondences: speculation and investigations," IEEE Trans. sign methodology., vol. 40 no. 10, pp. forty eight-fifty 3, Oct. 2004.

6. S. Haykin, M. Sellathurai, Y. de Jong, and T. Willink, "brief MIMO for some distance off correspondences," IEEE Commun. mag., vol. forty two, no. 10, pp. forty eight-53, Oct. 2004

7. ok. k. Wong, A. Paulraj, and R. D. Murch, "Efficient global elegance deciphering for over-load MIMO radio rope systems," IEEE Trans. far off Commun., vol. 6, no. 5, pp. 1833-1842, likewise can 2007.

8. N. Prasad, okay. Y. Kalbat, and X. Wang, "preferably efficient max-log APP demodulation in MIMO 
structures," IEEE J. Sel. topics sign. technique., vol. 5, no. eight, pp. 1400-1414, Sept. 2011.

9. S. supply and J. Cavers, "Execution development through joint revelation of cochannel sign utilizing wonderful assortment well-knownshows," IEEE Trans. Commun., vol. forty six, no. 8, pp. 1038-1049, Aug. 1998.

10. H. Murata and S. Yoshida, "Trellis-coded co-channel impedance canceler for little scale mobile radio," IEEE Trans. Commun., vol. forty five, pp. 1088-1094, Sept. 1997.

11. real channels and exchange, 3GPP TS 36.211 V11.five.zero, Jan. 2014.

12. P. Robertson, E. Villebrun, and P. Hoeher, "A test of satisfactory and doubtful MAP deciphering figurings jogging inside the log space," IEEE Int. Conf. Commun., vol. 2, pp. 1009-1013, Jun. 1995.

13. P. Chevillat and E. Eleftheriou, "Unraveling of trellis-encoded banner interior seeing intersymbol deterrent and hullabaloo," IEEE Trans. Commun., vol. 37, no. 7, pp. 669-676, Jul. 1989

14. S. enough. Soni, P. S. Chauhan, adequate. Vasudevan, and Y. Shanker, "faster interpreting in ISI channels," Int. Conf. rising enhancements in Networks and workstation Commun., pp. ninety eight-103, Apr. 2011.

15. F. Schuh, A. Schenk, and J. B. Huber, "Composed decoding for punctured convolutional encoded transmission over ISI-channels," Int. ITG Conf. Syst., Commun., and Coding, pp. 1-5, Jan. 2013.

16. F. Schuh, A. Schenk, and J. B. Huber, "faded eccentrics super trellis decoding for convolutionaly encoded transmission over ISI-channels," Int. Conf. Comp. systems the executives Commun., pp. 484-489, Jan. 2013.

17. M. Breiling and L. Hanzo, "The fantastic trellis sort of faster codes," IEEE Trans. suggest. concept, vol. 46, no. 6 , pp. 2212-2228, Sept. 2000.

18. W. Zhang, C. D'Amours, and A. Yongacoglu, "Trellis coded dependability plan for multi-customer systems on AWGN channels," IEEE Vehic. Tech. Conf., vol. 3, pp. 1722-1726, can likewise 2004.

19. Radio transmission and get-collectively, 3GPP TS 45.0.five V11.four.0, Jan. 2014.

20. o.k.. Li and X. Wang, "withdraw plot evaluation of faster multiuser territory," IEEE Trans. distant., vol. four, no. 1, pp. three hundred 311, Jan. 2005 